\title{
Profile of angle closure glaucoma in the Gangetic Delta
}

\author{
Dr. Chandrima Paul, Prof. Himadri Datta, Prof. Gautam Bhaduri \\ Regional Institute of Ophthalmology Medical College and Hospitals, Kolkata
}

\begin{abstract}
Purpose: To study the clinical profile of the types of primary angle closure patients in a rural and urban population in Eastern India.

Materials and Methods: International Society of Geographical and Epidemiological Ophthalmology (ISGEO) classification scheme was used to categorize patients. Clinic records of patients diagnosed as primary angle closure were reviewed. Clinical data including prior management was collected and analyzed. Main Outcome measures were age, sex, best corrected visual acuity (BCVA), intraocular pressure (IOP), gonioscopy, optic disc assessment and Frequency Doubling Perimetry(FDT). Logistic regression model and receiver operating curve (ROC) were calculated for predictors of type of glaucoma.

Results: 1904 eyes; males:982, females: 922) were diagnosed to have various subtypes of angle closure. Mean $( \pm S D)$ age at presentation was significantly higher for males (56.54 \pm 11.51 years) as compared to females $(52.76 \pm 10.46$ years $)(P<001)$. Primary angle closure glaucoma $(P A C G)$ was most frequently diagnosed subtype (45.24\%) followed by Primary angle closure (PAC) (40.46\%) and Primary angle closure suspect $(P A C S)(14.30 \%)$ respectively. The three subtypes differed significantly among their mean IOP (on ANOVA, $F$ $=12.04 ; P<001) . R O C$ yielded a very high AUC of 0.96 with strong discriminatory ability for PACG.

Conclusion: In our population based study, the significant predictors for the outcome of PACG included male gender, diminution of vision, the presence of pain and worsening grades of BCVA. Nearly half of PACG presented with advanced disease.
\end{abstract}

Keywords: Angle closure, blindness, epidemiology, glaucoma, Gangetic Delta, primary angle closure, primary angle closure suspect, primary angle closure glaucoma

\section{Introduction}

Glaucoma is the highest cause of irreversible blindness Worldwide,.Asians represent $47 \%$ of those with glaucoma and $87 \%$ of those with angle closure glaucoma (ACD) ${ }^{1}$. Primary angle closure glaucoma (PACG) has been reported to be more prevalent in South East Asian countries than the rest of the world ${ }^{2}$.This was a retrospective study to analyze the clinical aspects of all forms of angle closure glaucoma in patients from a population based survey in the Metropolitan city of Kolkata and Hooghly district in rural West Bengal.

\section{Materials and Methods}

The rural study area consists of 28 contiguous villages from the district of Hugli in West Bengal which are about 20kms surrounding the Rural base hospital located at Dhobapara,in Village Bakulia of this District.

Kolkata city, our urban study area, is about $100 \mathrm{kms}$ from the Rural base hospital at Bakulia. Kolkata stands on the Eastern Bank of River Ganga. The tail end of river Ganga flows by the side of Kolkata before it reaches Bay of Bengal about $180 \mathrm{Km}$. down stream from Kolkata. Kolkata is situated at the longitude of $88^{\circ} 30^{\prime} \mathrm{E}-22^{\circ} 33^{\prime} \mathrm{N}$ and an Altitude of 6.4 meters from sea level

The Area is 1480 sq. km. and divided into :

Old Kolkata: Sutanuti- Chitpur, Baghbazar, Sobhabazar \& Hatkhola. Kolkata - Dharmatala, Bowbazar, Simla, Janbazar. Gobindapur - Hastings, Maidan \& Bhowanipur

New Kolkata: North - Sinthi, Cossipore \& Gughudanga South -Tollygunge, Khidderpore \& Behala East - Salt Lake, Beliaghata \& Topsia. West - Hooghly river.

Greater Kolkata: Baruipur to Bansberia \& Kalyani to Budge Budge.

The Urban Study Sample consisted of Clusters from all three areas.

A three year retrospective analysis (January 2010-December2012) of the records of 8304 patients was done. Patients found to have angle closure were classified using International Society of Geographical and Epidemiological Ophthalmology (ISGEO) Classification ${ }^{2}$ - Approval was obtained from Institutional review board for retrospective data review, analysis and publication. Clinical records were reviewed in detail with respect to presenting complaints, best corrected visual acuity (BCVA), intra ocular pressure (IOP) (by Goldmann applanation tonometer), gonioscopy (using Zeiss 4-mirror goniolens), optic nerve head evaluation and FDP using C-20 field analysis (Humphrey Instruments Inc., San Leandro, CA). Grading used for gonioscopy was based on structures actually visualized. . 
Patients with incomplete records and secondary angle closure, such as lens-induced glaucoma, neovascular glaucoma, or uveitis, were specifically excluded.

\section{Patients were classified into:}

Primary angle closure suspects (PACS) if an appositional contact was present between the peripheral iris and posterior trabecular meshwork and more than 270 degree of posterior trabecular meshwork could not be visualized ${ }^{2}$.

Primary angle closure (PAC) patients had an eye with occludable drainage angle i.e., the posterior (usually pigmented) trabecular meshwork is seen for less than $90^{\circ}$ of angle circumference and features indicating that trabecular obstruction by peripheral iris has occurred, such as peripheral anterior synechiae, elevated IOP, iris whorling, "glaucomflecken" lens opacities or excessive pigment deposition on the trabecular surface, with no optic nerve head changes. ${ }^{1}$

Primary angle closure glaucoma (PACG) was labeled if disc and field changes were present with PAC (appositional or synechial) as defined above i.e., a vertical cup to disc ratio (VCDR) of 0.7 or greater or asymmetry between the right and left VCDRs of 0.2 or more, and a visual field defect consistent with glaucoma ${ }^{2}$. If the media opacities obscured optic disc assessment, then an IOP greater than $26 \mathrm{~mm}^{\mathrm{Hg}}$ and visual acuity worse than $20 / 400$, or evidence of previous glaucoma filtering surgery was considered. The VCDR and IOP criteria described above were based on the $97.5^{\text {th }}$ and $99.5^{\text {th }}$ percentiles for "hypernormals" in surveys described by Foster $e t a l^{2}$.

Minimal criteria for labeling a glaucomatous visual field defect were as follows: Glaucoma hemifield test (GHT) outside normal limits, pattern standard deviation (PSD) with $P$ values $<5 \%$, or a cluster of three or more points in the pattern deviation plot in a single hemifield with $P$ values $<5 \%$, one of which must have a $P$ value $<1 \%$. Any one of the preceding criteria, if found again on repeat testing on two tests within one month, was considered sufficient evidence of a glaucomatous visual field defect ${ }^{3,4}$. Visual fields were done for patients with BCVA of 20/200 or better. Advanced field defects were defined as mean deviation greater than $-12 \mathrm{~dB}$ and on Pattern deviation plot, points below 5\% between 37 to 55 with points below $1 \%$ ranging from 19 to 36 . The diagnosis was confirmed by either of the two senior consultants in all cases. We present our data using both the visual acuity and visual field criteria [World Health Organization (WHO) criteria] ${ }^{5}$ Eyes with advanced field damage and/or VCDR of more than 0.80 and/or BCVA less than 20/400 due to glaucomatous disc damage were classified as advanced glaucoma ${ }^{6}$

Statistical analysis was performed using SPSS statistical software $(15.0$ version). Data were descriptively analyzed for estimates of quantitative variables using mean and $95 \%$ confidence intervals. Chisquare test of independence was used to evaluate associations between qualitative variables and Glaucoma Classification groups. ANOVA was used to compare intraocular pressures measured in the eyes affected by three different types of glaucoma ( for 1904 eyes).All tests were two-tailed and $P$-values $<0.05$ were taken as significant.

\section{Results}

Of the 1904 eyes seen, since PACS and PAC do not have structural abnormality, the net proportion of PACG was $45.24 \%$.Amongst the angle closure subtypes, Primary angle closure glaucoma (PACG) was most frequently diagnosed subtype $(45.24 \%)$ followed by Primary angle closure (PAC) $(40.46 \%)$ and Primary angle closure suspect (PACS) (14.30\%).In patients with different subtypes of angle closure in both eyes, the eye with the higher degree of angle closure was used for categorization. The overall mean age at presentation for males was 58.60 years ( $95 \%$ CI; 56.80-60.40); significantly higher when compared to mean age of 54.40 years $(95 \%$ CI; 52.20-56.60) in females $(P<0.001)$. The mean age of presentation also varied significantly among patients stratified by types of glaucoma [Table 1]. However, on general linear model analysis using age as dependent variable and gender as well as type of glaucoma as fixed covariates, there was no significant group interaction between gender versus type of glaucoma $(\mathrm{F}=1.57 ; P=0.32)$ as far as mean age of patients was concerned

Amongst PACS, 86\% (192/224) had BCVA between 20/20 to 20/50 while latter was observed only in $72 \%$ (554/770) of PAC and $31.5 \%$ (239/761) of PACG patients.

Though patients with PACS, PAC and PACG differed among their mean IOP (on repeated measure ANOVA, $\mathrm{F}=24.35 ; P<0.001$.), they had no significant interaction with diagnostic categories of glaucoma as far as their mean IOP values are concerned. The blindness was attributed to advanced glaucomatous optic neuropathy.

Patients with diminution of vision had over 10 times the odds of developing PACG as compared to patients with PACS $(P<0.001$; OR $=14.21 ; 95 \%$ CI: 8.36-26.71). Similarly, diminution of vision increased over six times the odds of PACG over PAC group $(P<0.001$; OR: 8.32 ; 95\%CI: 6.86-16.85). The presence of pain also was a predictor of severity of glaucoma; odds higher for PACG in patients with pain as compared to PACS (4.18; 95\% CI: 2.13-6.71) as well as PAC patients (3.14; 95\% CI: 2.88- 5.41) respectively. Odds of colored 
haloes favored PACS (OR 4.41; 95\% CI $=1.90-8.43 ; P<0.001)$ as well as PAC $(\mathrm{OR} 3.93 ; 95 \% \mathrm{CI}=2.70-6.05$, $P<0.001)$ over the odds of PACG Receiver operating curve (ROC) yielded a very high area under curve (AUC) of 0.96 with strong discriminatory ability for PACG [Fig.1 ].

\section{Discussion}

Four population-based studies have been conducted in South India ${ }^{7-10}$ and one in West Bengal ${ }^{11}$ as regards prevalence of glaucoma. The results of these studies provide data about the magnitude of the problem. ${ }^{10}$ The aim of this study was to ascertain the prevalence and profile of various subtypes of ACG that present in East India.

We found PACG to be the most common type seen in $45.24 \%$. followed by PAC in $40.46 \%$. Amongst other studies, only CGS and West Bengal Glaucoma Study used ISGEO classification. In CGS, the prevalence of PACG, PAC and PACS was $0.9 \%, 0.71 \%$ and $6.3 \%$, respectively. The West Bengal study was limited to a rural population and a small sample size .

It is important for an ophthalmologist to identify signs of angle closure as, in India, asymptomatic chronic ACG mimicking POAG is common.Laser Peripheral Iridotomy (LPI) should be considered for all PAC, PACG and PACS patients.

\section{Conclusion:}

In our population based study, PACG was the most common type seen in $45.24 \%$. followed by PAC and finally PACS ,the significant predictors for the outcome of PACG included male gender, diminution of vision, the presence of pain and worsening grades of BCVA. Laser Peripheral Iridotomy (LPI) should be considered for all PAC, PACG and PACS patients.

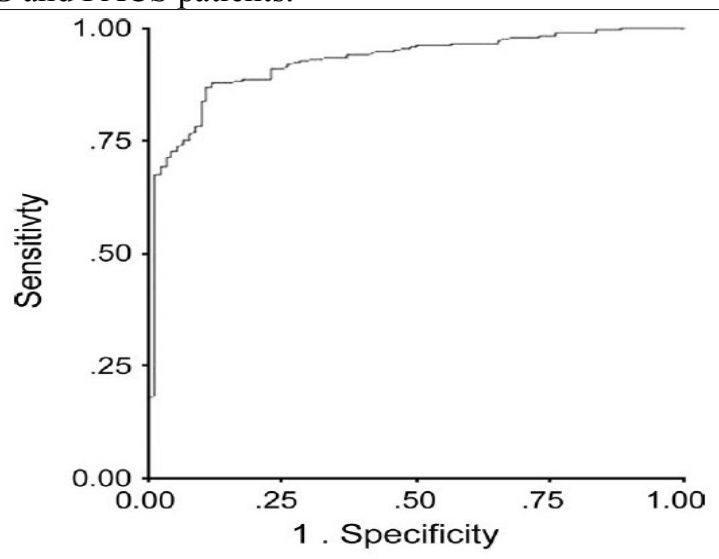

Fig 1 Receiver operating curve showing area under curve (0.96) for outcome of primary angle closure glaucoma

Table 1

Comparative analysis of quantitative variables of age and IOP stratified by Glaucoma subtypes by ANOVA

\begin{tabular}{|l|l|l|l|l|l|l|}
\hline & PACS (n=192) & PAC (n=554) & PACG (n=239) & F-value & P-value & Posthoc Tukey \\
\hline & & & & & & \\
\hline & Mean (95\%CI) & Mean (95\%CI) & Mean (95\%CI) & & & \\
\hline Age in years & 46.47 & 52.25 & 59.22 & 32.13 & $<.001$ & PACG $>$ PAC \\
\hline & $(44.42-48.53)$ & $(51.18-53.53)$ & $(58.00-60.22)$ & & & PACG $>$ PAC \\
\hline & & & & & & PAC $>$ PACS \\
\hline Eyes assessed & 224 & 770 & 761 & & & \\
\hline IOP & PACS & PAC & PACG & F-value* & $P$-value & \\
\hline & $17.32(16.22-18.54)$ & $18.24(17.62-19.86)$ & $22.02(21.04-23.06)$ & 31.17 & $<.001$ & PACG $>$ PAC \\
\hline & & & & & & PACG $>$ PACS \\
\hline & & & & & PAC $>$ PACS \\
\hline
\end{tabular}

\section{References}

[1]. Parul Ichhpujani, Surinder S Pandav, Aparna Ramasubramanian, and Sushmita Kaushik Indian J Ophthalmol. 2010 May-Jun; 58(3): 199-203. Profile of angle closure in a tertiary care center in north India

[2]. Foster PJ, Buhrmann R, Quigley HA, Johnson GJ. The definition and classification of glaucoma in prevalence surveys. Br J Ophthalmol. 2002;86:238-4. [PMC free article] [PubMed]

[3]. Hodapp E, Parrish RK, 2nd, Anderson DR. Clinical Decisions in Glaucoma. St Louis: Mosby Year Book Inc; 1993. pp. 52-9.

[4]. Anderson DR, editor. Automated Static Perimetry 123. St Louis: Mosby Year Book; 1992.

[5]. Tenth Revision. Vol. 1. Geneva: World Health Organization; 1992. International Statistical Classification of Diseases and Related Health Problems; pp. 456-7. 
[6]. Congdon N, Wang F, Tielsch JM. Issues in the epidemiology and population based screening of primary angle closure glaucoma. Surv Ophthalmol. 1992;36:411-23. [PubMed]

[7]. Thomas R, Garudadri C, Mandal A, Kumar R. Glaucoma in southern India. Ophthalmology. 2004;111:846. [PubMed]

[8]. Ramakrishnan R, Nirmalan PK, Krishnadas R, Thulasiraj RD, Tielsch JM, Katz J, et al. Glaucoma in a rural population of southern India: The Aravind comprehensive eye survey. Ophthalmology. 2003;110:1484-90.

[9]. Dandona L, Dandona R, Mandal P, Srinivas M, John RK, McCarty CA, Rao GN. Angle-closure glaucoma in an urban population in southern India. The Andhra Pradesh eye disease study. Ophthalmology. 2000;107:1710-6.

[10]. Vijaya L, George R, Arvind H, Baskaran M, Paul PG, Ramesh SV, Raju P, Kumaramanickavel G, Mccarty C. prevalence of angleclosure disease in a rural southern indian population. arch ophthalmol. 2006;124:403-9.

[11]. Raychaudhuri A, Lahiri SK, Bandyopadhyay M, Foster PJ, Reeves BC, Johnson GJ. A population based survey of the prevalence and types of glaucoma in rural West Bengal: The West Bengal Glaucoma Study. Br J Ophthalmol. 2005;89:1559-64. 